CERN-TH-97-187

PSU-TH-188

\title{
Orbits of Exceptional Groups, Duality and BPS States in String Theory
}

\author{
Sergio Ferrara円 and Murat Günaydin 9 \\ Theory Division \\ CERN \\ CH-1211 Geneva 23
}

\begin{abstract}
We give an invariant classification of orbits of the fundamental representations of exceptional groups $E_{7(7)}$ and $E_{6(6)}$ which classify BPS states in string and $\mathrm{M}$ theories toroidally compactified to $d=4$ and 5 . The exceptional Jordan algebra and the exceptional Freudenthal triple system and their cubic and quartic invariants play a major role in this classification. The cubic and quartic invariants correspond to the black hole entropy in $d=5$ and $d=4$, respectively. The classification of BPS states preserving different numbers of supersymmetries is in close parallel to the classification of the little groups and the orbits of timelike, lightlike and space-like vectors in Minkowski space. The orbits of BPS black holes in $N=2$ Maxwell-Einstein supergravity theories in $d=4$ and $d=5$ with symmetric space geometries are also classified including the exceptional $N=2$ theory that has $E_{7(-25)}$ and $E_{6(-26)}$ as its symmety in the respective dimensions.
\end{abstract}

\footnotetext{
${ }^{1}$ Work supported in part by EEC under TMR contract ERBFMRX-CT96-00 (LNF Frascati, INFN, Italy) and DOE grant DE-FGO3-91ER40662, DE-FGO2-96ER40559 e-mail: ferraras@vxcern.cern.ch

${ }^{2}$ Work supported in part by the National Science Foundation under Grant Number PHY-9631332.

Permanent address: Penn State University, Physics Dept. University Park, PA 16802

e-mail: murat@phys.psu.edu
} 


\section{Introduction}

The exceptional groups $E_{7(7)}$ and $E_{6(6)}$ appear as duality symmetries [1], 目] of the low energy actions and their discrete subgroups as symmetries of the non-perturbative BPS spectrum of string and M theories in $d=4$ and 5 preserving $N=8$ supersymmetry [3]. The charges of the extremal BPS black holes can be assigned to the fundamental representations of the exceptional groups $E_{7(7)}$ and $E_{6(6)}$ which are 56 and 27 dimensional ,respectively. The entropy of these black holes in $d=5$ and $d=4$ is given by the square root of the cubic and quartic invariants of $E_{6(6)}$ and $E_{7(7)}$, respectively [4, 5]. However, the charge configurations must satisfy additional restrictions depending on the number of supersymmetries preserved. In fact, the eigenvalues of the central charge matrix must be degenerate when more than one supersymmetry is preserved by the black hole solution. These constraints were recently investigated in terms of a certain set of invariant conditions on the representation [6]. In this paper we give a classification of such BPS states in terms of orbits of $E_{6(6)}$ and $E_{7(7)}$ in the corresponding representation. We then extend our results to $N=2$ Maxwell-Einstein supergravity theories (MESGT) in $d=5$ and $d=4$ dimensions whose scalar manifolds are symmetric spaces and determine the orbits of their BPS black holes. In particular we give the orbits of the exceptional $N=2$ MESGT that has $E_{6(-26)}$ and $E_{7(-25)}$ as its symmetry in five and four dimensions, respectively.

\section{Jordan Algebras, Exceptional Groups and Their Orbits}

The cubic invariant $I_{3}$ in the 27 dimensional representation of $E_{6}$ can be identified with the cubic norm of the exceptional Jordan algebra $J_{3}^{\mathbf{O}}$ of $3 \times 3$ hermitian matrices over the composition algebra of octonions $\mathbf{O}$ with the symmetric Jordan product

$$
j_{1} \circ j_{2}=j_{2} \circ j_{1}
$$

that satisfies the Jordan identity [7, 8, 9, 10, 11, 12]

$$
j_{1} \circ\left(j_{2} \circ j_{1}^{2}\right)=\left(j_{1} \circ j_{2}\right) \circ j_{1}^{2}
$$


A generic element $j$ of $J_{3}^{\mathbf{O}}$ has the form

$$
j=\left(\begin{array}{ccc}
\alpha_{1} & o_{3} & o_{2}^{*} \\
o_{3}^{*} & \alpha_{2} & o_{1} \\
o_{2} & o_{1}^{*} & \alpha_{3}
\end{array}\right)
$$

where $\alpha_{i}$ take values over the underlying field which we take to be real numbers $\mathbf{R}$ and $o_{i}(i=1,2,3)$ are elements of $\mathbf{O}$. The norm of an octonion $o$ is defined as

$$
N(o)=o o^{*}=o^{*} o
$$

where $*$ denotes octonion conjugation. There are different forms of the composition algebra of octonions. For the division algebra of real octonions the norm is invariant under $O(8)$ and for split octonions the norm is invariant under $O(4,4)$. For $N=8$ supergravity the relevant form of $J_{3}^{\mathbf{O}}$ is the one defined over the split octonions and for the exceptional $N=2$ MaxwellEinstein supergravity [11] it is the one defined over real octonions. We shall refer to the algebra defined over split octonions as the split exceptional Jordan algebra. [1 The automorphism group of the split exceptional Jordan algebra is the noncompact $F_{4(4)}$ with maximal compact subgroup $U S p(6) \times$ $U S p(2)$ [9]. Note that $F_{4(4)}$ is also the isometry group of the quaternionic manifold of a maximal $N=2$ matter-Einstein supergravity one can obtain by truncation of the $N=8$ supergravity in $d=5$ [11, 13]. The cubic norm $I_{3}$ of $J_{3}^{\mathbf{O}}$ is given by

$$
I_{3}=\alpha_{1} \alpha_{2} \alpha_{3}-\alpha_{1}\left(o_{1} o_{1}^{*}\right)-\alpha_{2}\left(o_{2} o_{2}^{*}\right)-\alpha_{3}\left(o_{3} o_{3}^{*}\right)+2 \operatorname{Re}\left(o_{1} o_{2} o_{3}\right)
$$

where $R e$ represents the real part of an octonion and satisfies

$$
\operatorname{Re}\left(o_{1} o_{2}\right) o_{3}=R e o_{1}\left(o_{2} o_{3}\right)
$$

The invariance group of the norm form of a Jordan algebra $J$ is referred to as the reduced structure group [8] and denoted as $S t_{0}(J)$. For the split exceptional Jordan algebra the reduced structure group is the exceptional group $E_{6(6)}$ with a maximal compact subgroup $U S p(8)$. We should also note that $U S p(8)$ is the automorphism group of the $N=8$ Poincare supersymmetry algebra in $d=5$ [2]. An element of $J_{3}^{\mathbf{O}}$ can be brought to a diagonal

\footnotetext{
${ }^{3}$ We should note that the split exceptional Jordan algebra and its associated symmetries first appeared in physics literature in attempts to find octonionic realizations of space-time supersymmetry 9.
} 
form by an $F_{4(4)}$ rotation [10] and if we denote the eigenvalues of a generic element $j$ as $\lambda_{i}(i=1,2,3)$ the cubic norm is simply

$$
I_{3}(j)=\lambda_{1} \lambda_{2} \lambda_{3}
$$

To make the analysis that follows clearer from a physics perspective we shall make an analogy with Minkowski space $M_{4}$ and its symmetries following [9, 14, 15]. A four vector in $M_{4}$ can be represented by $2 \times 2$ matrices $x=x_{\mu} \sigma^{\mu}$ where $\sigma^{0}=1_{2}$ and $\sigma^{i}(i=1,2,3)$ are the Pauli matrices. As $2 \times 2$ matrices the coordinates $x$ can be considered as elements of the Jordan algebra $J_{2}^{\mathbf{C}}$ of Hermitian matrices over the complex numbers $\mathbf{C}$ with the symmetric Jordan product which preserves hermiticity. The automorphism group of $J_{2}^{\mathbf{C}}$ is the covering group $S U(2)$ of the rotation group which is the analog of $F_{4(4)}$ for $J_{3}^{\mathbf{O}}$. The norm form of $J_{2}^{\mathbf{C}}$ is quadratic and is given by the ordinary determinant. The invariance group the quadratic norm of $J_{2}^{\mathbf{C}}$ is the covering group $S l(2, \mathbf{C})$ of the Lorentz group $S O(3,1)$ which is the analog of $E_{6(6)}$ for $J_{3}^{\mathrm{O}}$. In Minkowski space a vector is characterized by its norm and the parameters of the corresponding orbits. Time-like, spacelike and light-like vectors corresponding to positive, negative and vanishing norms have orbits $\frac{S l(2, \mathbf{C})}{S U(2)}, \frac{S l(2, \mathbf{C})}{S U(1,1)}$ and $\frac{S l(2, \mathbf{C})}{E_{2}}$, respectively. Similarly, we can characterize the elements of $J_{3}^{\mathbf{O}}$ by their norms and the parameters of their orbits. The generic orbit corresponding to a non-vanishing norm $I_{3}(j)$ has the 26 dimensional orbit

$$
\frac{E_{6(6)}}{F_{4(4)}}
$$

In contrast to the Minkowskian case the little groups of "space-like" and "time-like" vectors are the same in the case of $J_{3}^{\mathrm{O}}$ since its norm is cubic. 7 As for "light-like" elements $j$ of $J_{3}^{\mathrm{O}}$ with $I_{3}(j)=0$ there exist two distinct orbits depending on whether one or two of the eigenvalues of $j$ vanish. The generic light-like orbit corresponding to a single vanishing eigenvalue is given by the 26 dimensional coset space

$$
\frac{E_{6(6)}}{O(5,4) \odot T_{16}}
$$

where $\odot$ stands for semidirect product and $T_{16}$ are translations corresponding to the spinor representation of $O(5,4)$. The $T_{16}$ decomposes as $T_{8_{s}} \oplus T_{8_{c}}$

\footnotetext{
${ }^{4}$ As we shall see later the orbits of "time-like" and "space-like" vectors are quite different in four dimensions where the invariant norm form is quartic!
} 
under the $O(4,4)$ subgroup where $8_{c}$ and $8_{s}$ are the two spinor representations of $O(4,4)$.

The critical light-like orbit corresponds to an element $j$ with two vanishing eigenvalues and is given by the 17 dimensional space

$$
\frac{E_{6(6)}}{O(5,5) \odot T_{16}}
$$

where $T_{16}$ is an Abelian subgroup corresponding to the spinor representation of $O(5,5)$. We should note that the distinction between generic and critical light-like orbits does not exist in the Minkowskian case since the norm is quadratic in that case.

As is well-known the invariance group of the light-cone in Minkowski space $M_{4}$ is the conformal group $S O(4,2)$ which acts non-linearly. In fact the Minkowski space $M_{4}$ is simply the quotient space

$$
\frac{S O(4,2)}{[S O(3,1) \times O(1,1)] \odot T_{4}}
$$

When we represent the coordinates of $M_{4}$ in terms of hermitian $2 \times 2$ matrices the action of the conformal group can be represented as a linear fractional group. As such the conformal group can be interpreted as the linear fractional group of quaternions [16]. However, if we think of the $2 \times 2$ hermitian matrices as elements of the Jordan algebra $J_{2}^{\mathbf{C}}$ the conformal group becomes the linear fractional group of Jordan algebra $J_{2}^{\mathrm{C}}$ [17, 9, 14 which generalizes to all Jordan algebras and Jordan superalgebras 17, 14, 15]. The invariance group of the light-cone of $J_{3}^{\mathbf{O}}$ defined by the condition $I_{3}(j)=0$ is the noncompact exceptional group $E_{7(7)}$ which acts as the linear fractional group of $J_{3}^{\mathbf{O}}\left[17,9\right.$, 15]. This implies that the 27 dimensional space of $J_{3}^{\mathbf{O}}$ can be regarded as the quotient space

$$
\frac{E_{7(7)}}{\left[E_{6(6)} \times O(1,1)\right] \odot T_{27}}
$$

The above examples of linear fractional group actions are particular cases of the general nonlinear actions of noncompact groups $G$ whose Lie algebras $g$ admit a three grading with respect to a maximal rank subalgebra $g^{0}$

$$
g=g^{-1} \oplus g^{0} \oplus g^{+1}
$$

\footnotetext{
${ }^{5}$ In $d=4$ where the cubic norm is replaced by a quartic norm an even richer structure exists as we shall see later.
} 
In such cases there exists a nonlinear action of $G$ on the grade +1 space $g^{+1}$ via fractional linear transformations [17, 18]. In the case of $E_{7(7)}, g^{0}$ is simply the Lie algebra of $E_{6(6)} \times O(1,1)$ and $g^{+1}$ is the 27 dimensional subspace corresponding to $J_{3}^{\mathrm{O}}$. We will comment on the relevance of the "conformal" extensions of duality groups later.

We now consider the symmetries of superstring or $\mathrm{M}$ theories toroidally compactified to four dimensions with $N=8$ supersymmetry. In this case the duality group is $E_{7(7)}$ with maximal compact subgroup $S U(8)$. The compact subgroup $S U(8)$ acts as the automorphism group of the $N=8$ supersymmetry algebra. The generic charged vector for a BPS state has 56 components with a quartic norm $I_{4}$. The 56 dimensional representation space of $E_{7(7)}$ can be represented as elements of the exceptional Freudenthal triple system [19] which can be realized as $2 \times 2$ "matrices" of the form [20]:

$$
q=\left(\begin{array}{ll}
\alpha & x \\
y & \beta
\end{array}\right)
$$

where $\alpha, \beta \in \mathbf{R}$ and $x, y$ are elements of $J_{3}^{\mathbf{O}}$. One can define a symmetric four-linear form over the exceptional Freudenthal system which induces a quartic norm. Up to an overall normalization the quartic norm can be written as 20]

$$
I_{4}(q)=\{\alpha \beta-T(x, y)\}^{2}+6\left\{\alpha I_{3}(y)+\beta I_{3}(x)-T\left(x^{\#}, y^{\#}\right)\right\}
$$

where $T(x, y) \equiv \operatorname{Trace}(x \circ y)$ and \# stands for the quadratic adjoint map of $J_{3}^{\mathrm{O}}$ which has the property [21]

$$
x^{\# \#}=I_{3}(x) x
$$

The above quartic form $I_{4}(q)$ is invariant under the linear action of $E_{7(7)}$ on the exceptional Freudenthal triple system. The above realization of 56 of $E_{7(7)}$ corresponds to the decomposition

$$
56=27^{1}+\overline{27}^{-1}+1^{3}+\overline{1}^{-3}
$$

with respect to the $E_{6(6)} \times O(1,1)$ subgroup. We should also note that 56 can also be decomposed similarly with respect to the $E_{6(2)} \times U(1)$ subgroup of $E_{7(7)}$. In this case the two singlets are complex conjugates of each other carrying opposite charges with respect to $U(1) . E_{6(2)}$ has the maximal compact subgroup $S U(6) \times S U(2)$ and corresponds to the isometry group of the quaternionic manifold of a maximal $N=2$ matter-Einstein supergravity 
truncation of the $N=8$ supergravity in $d=4$ [11, 13]. In contrast to the five dimensional case and in analogy with the Minkowskian case we have two different classes of generic orbits with non-vanishing quartic form $I_{4}$. They correspond to

$$
\frac{E_{7(7)}}{E_{6(6)}}
$$

and to

$$
\frac{E_{7(7)}}{E_{6(2)}}
$$

As in [6] we choose the overall sign of the quartic invariant such that it corresponds to entropy of the BPS black holes. With this choice the orbit corresponding to $\frac{E_{7(7)}}{E_{6(6)}}$ has $I_{4}<0$ and the orbit corresponding to $\frac{E_{7(7)}}{E_{6(2)}}$ has $I_{4}>0$. This can be seen from the decomposition of 56 of $E_{7(7)}$ with respect to $S U(6) \times S U(2)$

$$
56=(15,1)+(\overline{15}, 1)+(6,2)+(\overline{6}, \overline{2})+1+\overline{1}
$$

and retaining the singlets [13].

We now consider "light-like orbits" for which $I_{4}=0$. There are 3 distinct cases depending on the number of vanishing eigenvalues that lead to vanishing $I_{4}$. We define the generic light-like orbit to be one for which a single eigenvalue vanishes. The orbit in this case is given by

$$
\frac{E_{7(7)}}{F_{4(4)} \odot T_{26}}
$$

where $T_{26}$ is a 26 dimensional Abelian subgroup of $E_{7(7)}$. The critical lightlike orbit has two vanishing eigenvalues and correspond to the 45 dimensional orbit

$$
\frac{E_{7(7)}}{O(6,5) \odot\left(T_{32} \oplus T_{1}\right)}
$$

The 88 dimensional triangular subgroup of $E_{7(7)}$ above is a subgroup of the better known triangular subgroup

$$
O(6,6) \odot\left(T_{32} \oplus T_{1}\right)
$$

of $E_{7(7)}$ [22, 24. The doubly critical light-like orbit with three vanishing eigenvalues is given by the 28 dimensional quotient space

$$
\frac{E_{7(7)}}{E_{6(6)} \odot T_{27}}
$$


We should note that the determination of the little groups that appear in the denominators of the above quotient spaces follows directly from the various symmetry groups of $J_{3}^{\mathrm{O}}$ and their different gradings [22, 23, 24]. In the next section we shall obtain the counting of the dimensions of the orbits via a complementary procedure that follows from the normal form for the central charge matrix and which relates orbits to BPS states preserving different number of supersymmetries.

\section{BPS States and Supersymmetry}

Extremal BPS black holes of $N=8$ supergravity correspond to massive representations of the $N=8$ supersymmetry algebra that saturate the BPS bound. They fall into three categories depending on whether the black hole background preserves $1 / 2,1 / 4$ or $1 / 8$ of the original supersymmetry [25]. BPS states preserving $1 / 8$ supersymmetry are the only ones with nonvanishing entropy and regular horizon. BPS states with $1 / 4$ and $1 / 2$ supersymmetry have vanishing entropy [5]. In this section we will relate the orbits of the fundamental representations of $E_{6(6)}$ and $E_{7(7)}$ to these different cases. To this end we will relate our classification to the analysis of [6]. The degeneracy of the eigenvalues of the central charge matrix was there related to U-duality invariant constraints on the central charge matrix. This analysis heavily depends on the so-called normal frame of the charge matrix which is generically obtained by making a rotation under the automorphism group of the supersymmetry algebra. The automorphism group of the supersymmetry algebra essentially coincides with the maximal compact subgroup of the duality group.

Let us first study the case of $d=5$. The 27 dimensional representation of $E_{6(6)}$ corresponds to the symplectic traceless anti-symmetric tensor representation of $U S p(8)$. It can be brought to a skew diagonal form via an $U S p(8)$ transformation. In terms of the eigenvalues $e_{i}$ of this matrix the cubic invariant takes the form [6]

$$
I_{3}=\left(e_{1}+e_{2}\right)\left(e_{1}+e_{3}\right)\left(e_{2}+e_{3}\right)
$$

We then see that the three different orbits described in the preceding section correspond to the following three cases [6]
a) $I_{3} \neq 0$
b) $I_{3}=0, \quad \frac{\partial I_{3}}{\partial e_{i}} \neq 0$
c) $\frac{\partial I_{3}}{\partial e_{i}}=0$ 
They correspond to the cases of $1 / 8,1 / 4$ and $1 / 2$ supersymmetry since in case a) all eigenvalues are different from zero ; in case b) two eigenvalues coincide and in case c) all three eigenvalues coincide. Let us now count the parameters of these 3 different orbits. We first note that the subgroup of $F_{4(4)}$ that preserves the normal form is $O(4,4)$ with maximal compact subgroup $S U(2)^{4}$. Thus the generic case of $I_{3} \neq 0$ involves 3 eigenvalues [26] plus 24 angles [27] corresponding to

$$
\frac{U S p(8)}{S U(2)^{4}}
$$

In case b) the little group is $O(5,4)$. This is a subgroup of the triangular subgroup $O(5,4) \odot T_{16}$ of $E_{6(6)}$ and again we have $2+24=26$ parameters. In case c) corresponding to the critical orbit the little group of the normal form is $O(5,5)$ with maximal compact subgroup $O(5) \times O(5)=U S p(4) \times$ $U S p(4)$. Thus the number of parameters is one eigenvalue plus 16 angles of $\frac{U S p(8)}{U S p(4) \times U S p(4)}$. Note that $O(5,5)$ is a subgroup of the triangular little group of the 17 dimensional orbit.

In $d=4$ the 56 dimensional representation of $E_{7(7)}$ can also be described by a complex $8 \times 8$ matrix and its complex conjugate. This $8 \times 8$ matrix can be brought to a skew diagonal form by an $S U(8)$ rotation. The skew diagonal form has 5 parameters [26], an overall phase and four real positive skew diagonal eigenvalues. The skew diagonal form is invariant under $O(4,4)$ which is the subgroup of $E_{6(2)}$ that preserves the normal form. The generic orbit then can be parametrized by five "normal" coordinates plus 51 angles [27 in $\frac{S U(8)}{U S p(2)^{4}}$. In four dimensions the extra condition for a generic state to be $1 / 8 \mathrm{BPS}$ with non-vanishing entropy is that $I_{4}>0$ [6]. This is a consequence of the fact that at the horizon all central charge eigenvalues but the BPS mass vanish [4]. Thus one has [4, 13]

$$
I_{4}=I_{4 \text { Horizon }}=M_{B P S(\text { Horizon })}^{4}
$$

This selects the "time-like" orbit $\frac{E_{7(7)}}{E_{6(2)}}$.

Let us now consider the 3 light-like orbits. The generic ( 55 dimensional) light-like orbit has four different eigenvalues of the $8 \times 8$ matrix and still preserves $1 / 8$ supersymmetry. The critical light-like orbit corresponding to $1 / 4$ supersymmetry has eigenvalues that coincide in pairs and zero overall phase [6]. The simple part of the little group in this case is $O(5,5)$ and the number of parameters is given by the two normal parameters plus the 43 
angles of $\frac{S U(8)}{U S p(4)^{2}}$. The double critical orbit corresponds to four coinciding eigenvalues in the normal form, zero phase and $1 / 2$ supersymmetry. The little group preserving this form is $E_{6(6)}$ with maximal compact subgroup $U S p(8)$. The total number of parameters of the double critical orbit is one normal parameter and 27 angles of $\frac{S U(8)}{U S p(8)}$ which agrees with the results of the previous section.

\section{The Orbits of BPS States of $N=2$ Maxwell- Einstein Supergravity Theories in Five and Four Dimensions}

In this section we shall extend our results to the classification of the orbits of $N=2$ Maxwell-Einstein supergravity theories (MESGT) in five and four space-time dimensions. The $N=2$ MESGT's in five dimensions were constructed by Günaydin, Sierra and Townsend (GST) sometime ago [11, 12, 28]. In the work of GST a complete characterization of the geometry of five dimensional $N=2$ MESGT's theories as well as the geometry of four dimensional theories that are obtained by dimensional reduction from five dimensions was given. The geometry of the corresponding four dimensional geometries came to be called a very special geometry to distinguish them from the special geometry of general $N=2$ theories in four dimensions [29]. Most of these coset spaces appear as moduli spaces of untwisted sectors of orbifold compactification of type II string theories , preserving $N=2 \mathrm{su}-$ persymmetry. For a related discussion we refer to reference [30]. The $N=2$ MESGT's in five dimensions and their geometries are characterized by a cubic norm form defined by the completely symmetric tensor $C_{I J K}$ that appears in front of the FFA type coupling term of the vector fields. When this cubic form is taken to be the norm form of a Jordan algebra of degree three, the scalar manifold of the corresponding $N=2$ theory is a symmetric space of the form $G / H$ where $G$ and $H$ are the reduced structure and automorphism groups of the underlying Jordan algebra, respectively [11, 12]. There is an infinite family of $N=2$ MESGT's defined by reducible Jordan algebras of degree three whose scalar manifolds $\mathcal{M}$ are

$$
\mathcal{M}=\frac{S O(n-1,1)}{S O(n-1)} \times S O(1,1)
$$

where $n \geq 1$ is the number of vector multiplets coupled to $N=2$ supergravity. Furthermore, there exist four $N=2$ MESGT's defined by four 
simple Jordan algebras of degree 3 with irreducible norm forms. They are the Jordan algebras of hermitian $3 \times 3$ matrices with over the four division algebras, namely the reals $\mathbf{R}$, complex numbers $\mathbf{C}$, quaternions $\mathbf{H}$ and the octonions $\mathbf{O}$. Their scalar manifolds are

$$
\frac{S L(3, \mathbf{R})}{S O(3)} ; \frac{S L(3, \mathbf{C})}{S U(3)} ; \frac{S U^{*}(6)}{U S p(6)} ; \frac{E_{6(-26)}}{F_{4}}
$$

These theories are referred to as "magical" MESGT's since their symmetry groups in $\mathrm{d}=5,4,3$ dimensions are the groups of the famous Magic Square of Freudenthal, Rozenfeld and Tits associated with some remarkable geometries 31. Except for the exceptional MESGT defined by the exceptional Jordan algebra $J_{3}^{\mathbf{O}}$ these theories can all be obtained by a consistent truncation of the $N=8$ supergravity in the respective dimensions [11]. In addition to the above there exist one other infinite family of $N=2$ MESGT's whose scalar manifolds are symmetric spaces of the form [28]

$$
\frac{S O(n, 1)}{S O(n)}
$$

For this family the cubic forms are, in general, not given by the norm forms of Jordan algebras of degree 3 [28].

The entropy of the BPS black holes in $N=2$ MESGT's studied so far in the literature is given by the square root of the modulus of the cubic invariant $I_{3}$ determined by the tensor $C_{I J K}$ and this is believed to hold in general 近, 32]. One can give a complete classification of the orbits of such states in the $N=2$ MESGT's listed above. For BPS states with $I_{3}$ such that all three eigenvalues are positive the orbit coincides with the scalar manifold $G / H$ of the MESGT. For BPS states with $I_{3}$ such that two of the eigenvalues are negative and one positive the orbits are of the form $G / \tilde{H}$ where $\tilde{H}$ is a different real form of $H$. We list the possible orbits in Table I.9

We should note that in $N=2$ MESGT's in $d=5$ the scalar manifold is a hypersurface defined by the condition that the cubic norm be equal to a nonzero positive constant which was chosen to be one [12]. Similarly, the metric of the kinetic energy term of the vector fields was given by a tensor evaluated at this hypersurface. Therefore, the points where the entropy vanishes are expected to correspond to phase transition points in these theories and/or

\footnotetext{
${ }^{6}$ We should note that the corresponding two cases in the $N=8$ supergravity lead to isomorphic orbits. Whether both of the distinct orbits in $N=2$ MESGT correspond to orbits of BPS states remains to be established.
} 


\begin{tabular}{|c|c|}
\hline$G / H$ & $G / \tilde{H}$ \\
\hline$\frac{S O(n-1,1)}{S O(n-1)} \times S O(1,1)$ & $\frac{S O(n-1,1)}{S O(n-2), 1)} \times S O(1,1)$ \\
$\frac{S L(3, \mathbf{R})}{S O(3)}$ & $\frac{S L(3, \mathbf{R})}{S L(2, \mathbf{R})}$ \\
$\frac{S L(3, \mathbf{C})}{S U(3)}$ & $\frac{S L(3, \mathbf{C})}{S U(2,1)}$ \\
$\frac{S U^{*}(6)}{U S p(6)}$ & $\frac{S U^{*}(6)}{S p(4,2)}$ \\
$\frac{E_{6(-26)}}{F_{4}}$ & $\frac{E_{6(-26)}}{F_{4(-20)}}$ \\
$\frac{S O(n, 1)}{S O(n)}$ & $\frac{S O(n, 1)}{S O(n-1,1)}$ \\
\hline
\end{tabular}

Table 1: Above we list the possible orbits of the $N=2$ MESGT's in $d=5$ whose scalar manifolds are symmetric spaces of the form $G / H$. The first column corresponds to orbits with nonvanishing cubic norm such that all three eigenvalues are positive and coincides with $G / H$. The second column lists orbits with positive cubic norm such that two of the eigenvalues are negative.

going to the boundary of their moduli spaces in a singular fashion [33]. At these points one expects the number of massless degrees of freedom to change as well. Here we shall give the orbits corresponding to vanishing cubic norm form for the exceptional $N=2$ MESGT in order to highlight the differences with the $N=8$ theory. The light-like orbit with a single vanishing eigenvalue such that the other two eigenvalues are positive is

$$
\frac{E_{6(-26)}}{O(9) \odot T_{16}}
$$

If the two non-vanishing eigenvalues have opposite signs then the lightlike orbit is

$$
\frac{E_{6(-26)}}{O(8,1) \odot T_{16}}
$$

For the $N=8$ theory the corresponding orbits turn out to be isomorphic. The critical light-like orbit for the exceptional theory with two vanishing eigenvalues is

$$
\frac{E_{6(-26)}}{O(9,1) \odot T_{16}}
$$

The four dimensional $N=2$ MESGT's whose scalar manifolds are symmetric spaces $G / H$ [34] can all be obtained from the five dimensional theories listed above by dimensional reduction [12, 28]. For the theories defined by 
the reducible Jordan algebras the scalar manifold in $d=4$ is given as [11, 12]:

$$
\frac{S O(n, 2)}{S O(n) \times S O(2)} \times \frac{S O(2,1)}{S O(2)}
$$

The scalar manifolds of the magical supergravity theories in $d=4$ are [11, 12]

$$
\frac{S p(6, \mathbf{R})}{U(3)} ; \frac{S U(3,3)}{S(U(3) \times U(3))} ; \frac{S O^{*}(12)}{U(6)} ; \frac{E_{7(-25)}}{E_{6} \times U(1)}
$$

and the scalar manifolds of the four dimensional $N=2$ theories corresponding to the family (4-6) are 28]

$$
\frac{S U(n, 1)}{U(n)}
$$

Note that the manifolds $G / H$ of scalar fields in four dimensions are special Kahler such that $H$ has an Abelian $U(1)$ factor $H=H_{0} \times U(1)$.

We recall that in $N=2$ MESGT's in $\mathrm{d}=4$ the entropy formula for arbitrary geometry can be written in a manifestly positive form as [4]:

$$
S=\left\{|Z|^{2}+\left|D_{i} Z\right|^{2}\right\}_{f i x}=-\left.\frac{1}{2} P^{T} M(\mathcal{N}) P\right|_{f i x}
$$

where $Z$ is the $N=2$ central charge and $D_{i} Z$ is the Kähler condensate. The subscript $(f i x)$ means that the quantities are computed for fixed moduli which extremize the BPS mass 朋. The $P=(p, q)$ is a $2 n+2$ dimensional vector of quantized charges and $M(\mathcal{N})$ is a $(2 n+2) \times(2 n+2)$ symplectic matrix constructed in terms of the symmetric $(n+1) \times(n+1)$ complex "kinetic' matrix $\mathcal{N}$ [1].

For those theories whose scalar manifolds are symmetric spaces $G / H$ the entropy reduces to a $G$ invariant in the corresponding representation $P$ of $G$. Specifically, the entropy of the BPS black holes in $\mathrm{d}=4$ is given by the square root of the quartic invariant $I_{4}$ of $G$ in the representation $P[\llbracket]$. For the family of $N=2$ theories with the scalar manifold $\frac{S U(n, 1)}{U(n)}$ the quartic form turns out to be the square of a quadratic form and the entropy is given by this quadratic function of electric and magnetic charges. Requiring that $I_{4}$ be positive for BPS black holes leads to two different types of orbits. For those $N=2$ MESGT's defined by Jordan algebras of degree three these two types of orbits are of the form

$$
\frac{G}{H_{0}}
$$




\begin{tabular}{|c|c|}
\hline$G / H_{0}$ & $G / \tilde{H}_{0}$ \\
\hline$\frac{S O(n, 2) \times S O(2,1)}{S O(n) \times S O(2)}$ & $\frac{S O(n, 2) \times S O(2,1)}{S O(n-1,1) \times S O(1,1)}$ \\
$\frac{S p(6, \mathbf{R})}{S U(3)}$ & $\frac{S p(6, \mathbf{R})}{S L(3, \mathbf{R})}$ \\
$\frac{S U(3,3)}{S U(3) \times S U(3)}$ & $\frac{S U(3,3)}{S L(3, \mathbf{C})}$ \\
$\frac{S O^{*}(12)}{S U(6)}$ & $\frac{S O^{*}(12)}{S U^{*}(6)}$ \\
$\frac{E_{7(-25)}}{E_{6}}$ & $\frac{E_{7(-25)}}{E_{6(-26)}}$ \\
$\frac{S U(n, 1)}{S U(n)}$ & $\frac{S U(n, 1)}{S U(n-1,1)}$ \\
\hline
\end{tabular}

Table 2: Above we list the possible orbits of the $N=2$ MESGT's in $d=4$ whose scalar manifolds are symmetric spaces of the form $G / H$. The first column corresponds to orbits with nonvanishing quartic form such that all four eigenvalues are positive. The second column lists orbits with positive quartic form such that two of the eigenvalues are negative.

and of the form

$$
\frac{G}{\tilde{H}_{0}}
$$

where $H_{0}$ is the maximal compact subgoup of $G$ with $U(1)$ factor deleted and $\tilde{H}_{0}$ is the noncompact real form of $H_{0}$ which is isomorphic to the noncompact symmetry group of the corresponding five dimensional theory. We list these orbits in Table II.

Again for comparison with the $N=8$ theory we will give the orbits for vanishing quartic form of the exceptional $N=2$ theory. We find two lightlike orbits:

$$
\begin{array}{r}
\frac{E_{7(-25)}}{F_{4} \odot T_{26}} \\
\frac{E_{7(-25)}}{F_{4(-20)} \odot T_{26}}
\end{array}
$$

and two critical lightlike orbits:

$$
\begin{gathered}
\frac{E_{7(-25)}}{O(9,2) \odot\left[T_{32} \oplus T_{1}\right]} \\
\frac{E_{7(-25)}}{O(10,1) \odot\left[T_{32} \oplus T_{1}\right]}
\end{gathered}
$$


as well as two doubly critical lightlike orbits:

$$
\begin{array}{r}
\frac{E_{7(-25)}}{E_{6(-26)} \odot T_{27}} \\
\frac{E_{7(-25)}}{E_{6} \odot T_{27}}
\end{array}
$$

\section{Conclusions}

In the first part of our paper we have determined the orbits of the exceptional groups corresponding to duality symmetries of toroidally compactified string or $\mathrm{M}$ theories to 4 and 5 dimensions. Our analysis is classical and for the quantum theory the relevant U-duality groups become discrete [3]. We expect our results can be extended to the discrete cases as well.

An intriguing aspect of our results is the appearance of larger symmetries acting non-linearly on the generalized light-cones defined by vanishing cubic and quartic forms. In $d=5$ this turns out to be $E_{7(7)}$ that acts via linear fractional transformations on $J_{3}^{\mathrm{O}}$. There is a a discrete subgroup of $E_{7(7)}$ that acts via discrete linear fractional transformations on $J_{3}^{\mathbf{O}}$. This makes it tempting to speculate that the generalized conformal group acts as spectrum generating symmetry of the string or $\mathrm{M}$ theory toroidally compactified to $d=5$. In four dimensions we expect the analog of this generalized conformal group to be $E_{8(8)}$. However, $E_{8(8)}$ does not admit 3 -grading with respect to any maximal rank subalgebra and hence does not act via linear fractional transformations on 56 of $E_{7(7)}$. However, it has a non-linear action on a 57 dimensional space which splits as $56+1$ under $E_{7(7)}$ 35]. The physical meaning of this extra singlet is not clear. This problem may be related to the difficulty in extending the results on BPS black holes to 3 dimensions. We should also note that our results can be extended to theories with less supersymmetry such as heterotic strings and to dualities in space-time dimensions greater than five.

In the latter part of our paper we extended our results to the classification of the orbits of $N=2$ MESGT's whose scalar manifolds are symmetric spaces in five and four dimensions. We find that different signs of charge eigenvalues lead to different orbits in this case. We also listed the orbits for vanishing entropy for the exceptional $N=2$ MESGT.

The complete list of orbits of $N=2$ MESGT's in $d=4$ and $d=5$ with vanishing entropy, their physical discussion and the extension of the $N=8$ 
results to higher dimensional theories as well as further details of the results presented here will be given elsewhere [36].

Acknowledgements: One of us (S.F) would like to thank C. Savoy for discussions regarding the material of section 3 and J. Maldacena for several discussions. The other (M.G) would like to thank H. Nicolai for numerous enlightening discussions.

\section{References}

[1] E. Cremmer and B. Julia, Nucl. Phys. B159 (1979) 141.

[2] E. Cremmer, in "Supergravity, ed. by S. Ferrara and J. Taylor, p 133.

[3] C. Hull and P.K. Townsend, Nucl. Phys. B438 (1995) 109.

[4] S. Ferrara and R. Kallosh, Phys. Rev. D54 (1996) 1514 and Phys. Rev. D54 (1996) 1525.

[5] R. Kallosh and B. Kol, Phys. Rev. D53 (1996) 5344.

[6] S. Ferrara and J. Maldacena, hep-th/9706097.

[7] P. Jordan, J. von Neumann and E. Wigner, Ann. Math. 36 (1934) 29.

[8] N. Jacobson, Ann. Math. Soc. Coll. Publ. 39 (1968).

[9] M. Günaydin, Nuovo Cimento 29 (1975) 467.

[10] M. Günaydin, C. Piron and H. Ruegg, Commun. Math. Phys. 61 (1978) 69.

[11] M. Günaydin, G. Sierra and P. K. Townsend, Phys. Lett. 133B (1983) 72 .

[12] M. Günaydin, G. Sierra and P. K. Townsend, Nucl. Phys. B242 (1984) 244; Nucl. Phys. B253 (1985) 573.

[13] L. Andrianopoli, R. D'Auria and S. Ferrara, hep-th/9703156, Phys. Lett. B403 (1997) 12 and hep-th/9705024, to appear in Physics Letters B.

[14] M. Günaydin, Ann. Israel Phys. Soc. 3(1980)279 ; Mod. Phys. Lett. A8 (1993) 1407-1416. 
[15] M. Günaydin, in "Elementary Particles and the Universe: Essays in Honor of Murray Gell-Mann", ed. by J.H. Schwarz, Cambridge University Press (1991) ; J. Math. Phys. 31 (1990) 1776.

[16] F. Gürsey, Nuovo Cimento 3 (1956) 988.

[17] M. Koecher, Amer. J. Math. 89(1967)787; "An elementary approach to bounded symmetric domains", Rice University Lecture Notes, Houston (1969).

[18] I. Bars and M. Günaydin, Commun. Math. Phys. 91 (1983) 31.

[19] H. Freudenthal, Adv. Math. 1 (1964)145.

[20] See J.R. Faulkner, Trans. Amer. Math. Soc. 167 (1972)49 and the references therein.

[21] K. McCrimmon, Trans. Amer. Math. Soc. 139(1969) 495.

[22] I.L. Kantor, Trudy Sem. Vector. Anal. 16 (1972)407.

[23] I. Bars and M. Günaydin, J. Math. Phys. 20 (1979) 1977.

[24] M. Günaydin and S. Hyun, Nucl. Phys. B373 (1992) 688;

M. Günaydin, Phys. Rev. D47 (1993) 3600.

[25] S. Ferrara, C. Savoy and B. Zumino, Phys. Lett. 100B (1981) 393.

[26] M. Cvetic and D. Youm, hep-th/9512127 ; M. Cvetic and A. Tseytlin, Phys. Rev. D53 (1995) 5412; M. Cvetic and I. Gaida, hep-th/9703134.

[27] M. Cvetic and C. Hull, Nucl. Phys. B480 (1996) 296-316 .

[28] M. Günaydin, G. Sierra and P.K. Townsend, Clas. Quant. Grav. 3 (1986) 763-771.

[29] See B. de Wit and A. van Proeyen, Commun. Math. Phys. 149 (1992) 307-334 and the references therein.

[30] S. Cecotti, S. Ferrara and L. Girardello, Int. Jour. Mod. Phys. 4 (1989) 2475 .

[31] H. Freudenthal, Proc. Konink.Ned. Akad. Wetenschap A62 (1959) 447; B.A. Rozenfeld, Dokl. Akad. Nauk. SSSR 106 (1956) 600 ; J. Tits, Mem. Acad. Roy. Belg. Sci. 29 (1955) fasc. 3. 
[32] S. Ferrara, R. Kallosh and A. Strominger, Phys. Rev. D52 (1995) 5412; A. Strominger, Phys. Lett. 383B (1996) 39 .

[33] A. Chou, R. Kallosh, J. Rahmfeld, S.-J. Rey, M. Shmakova, W.K. Wong, hep-th/9704142 ; S. Ferrara, G.W. Gibbons and R. Kallosh, hep-th/9702103.

[34] E. Cremmer and A. van Proeyen, Class. Quan. Grav. 2(1986) 763.

[35] B. Bina and M. Günaydin, hep-th/9703188, to appear in Nucl. Phys. B.

[36] S. Ferrara and M. Günaydin, in preparation. 